\title{
The high precision double slit device of the slit assembly for the FLEX instrument
}

\section{Matthias Mohaupt, Uwe Zeitner, Gerd Harnisch, Thomas Bolz, Stefan Risse, et al.}

Matthias Mohaupt, Uwe Zeitner, Gerd Harnisch, Thomas Bolz, Stefan Risse, Andreas Gebhardt, Henrik von Lukowicz, Thomas Peschel, Uwe Hübner, Yann Gerome, Markus Erhard, Gehard Huber, "The high precision double slit device of the slit assembly for the FLEX instrument," Proc. SPIE 11852, International Conference on Space Optics - ICSO 2020, 118520K (11 June 2021); doi: 10.1117/12.2599166

SPIE Event: International Conference on Space Optics - ICSO 2021, 2021, Online Only 


\section{International Conference on Space Optics-ICSO 2020}

Virtual Conference

30 March-2 April 2021

Edited by Bruno Cugny, Zoran Sodnik, and Nikos Karafolas
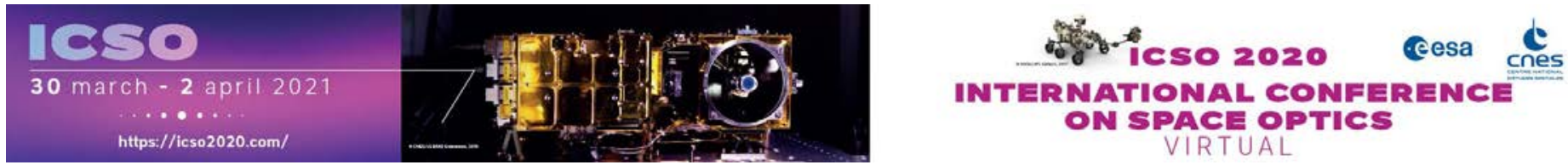

The high precision double slit device of the slit assembly for the FLEX instrument

\section{Cesa isoporecestings denes}




\title{
The high precision double slit device of the Slit assembly for the FLEX Instrument
}

\author{
Matthias Mohaupt ${ }^{1}$, Uwe Zeitner $^{1)}$, Gerd Harnisch ${ }^{1)}$, Thomas Bolz ${ }^{1)}$, Stefan Risse ${ }^{1)}$, Andreas \\ Gebhardt $^{1)}$, Henrik von Lukowicz ${ }^{1}$, Thomas Peschel ${ }^{1)}$, Uwe Hübner ${ }^{3)}$, Yann Gerome ${ }^{2)}$, Markus \\ Erhard $^{2}$, Gerhard Huber ${ }^{2)}$
}

1) Fraunhofer Institute for Applied Optics and Precision Engineering, Albert-Einstein-Strasse 7, D-07745 Jena, Germany

2) OHB System AG, Manfred-Fuchs-Strasse 1, D-82234 Weßling, Germany

3) Leibniz Institute of Photonic Technology, Albert-Einstein-Strasse 9, D-07745 Jena, Germany

\author{
Matthias.Mohaupt@iof.fraunhofer.de
}

\begin{abstract}
The high precision Slit Assembly is a key component of the FLEX instrument. Two different input beams for the Low- and the High-resolution spectrometer, respectively, will be generated by the Slit assembly. The paper presents design and implementation of its optical key components, which are a highly precise double slit and two mirrors achieving spatial channel separation and spectral filtering.

High alignment and stability requirements, as well as stringent envelope restrictions are driving the mechanical design of the Slit Assembly. We demonstrate solutions in design and manufacturing techniques as well as achieved performance under thermal and mechanical loads.

The main focus of the paper is on the development and realization of the double slit device. This device is designed to mount a silicon double slit chip into a mechanical holder providing mechanical and gauge interfaces to the slit assembly. The slit position is aligned to mechanical interfaces to meet the tight positioning requirements.

A dedicated lithographic structuring process chain was developed for the manufacturing of the double slit to fulfill a number of challenging requirements; i.e. the absolute slit width accuracy of less than $2 \mu \mathrm{m}$ peak to valley, and slit planarity of less than $10 \mu \mathrm{m}$ peak to valley. It is based on the adaptation of lithographic structuring techniques for etching of Silicon wafers. The overall manufacturing process is the result of an extended technology development phase. The manufactured slits are coated with a black coating layer to reach the specified optical reflectivity and the optical density.

The results of environmental tests of the Double slit device Breadboard, i.e. the thermal vacuum test, the shock test, and the vibration test, are discussed.
\end{abstract}

Keywords: Slit assembly, hyper spectral, high resolution spectrometer, low resolution spectrometer, double slit, assembly, integration, test, FLEX FLORIS fluorescence explorer, earth observation 


\section{INTRODUCTION}

The FLEX mission will map vegetation fluorescence to quantify photosynthetic activity. By quantifying vegetation fluorescence, the photochemical reflectance index, the surface temperature, the FLEX mission concept will provide the most innovative and unique set of measurements to increase our understanding of actual photosynthetic efficiency, and the status of vegetation health and plant performance, while addressing related societal challenges previously identified. [1]

FLEX instrument contains two spectrometers called High Resolution (HR) and Low Resolution (LR) spectrometer, respectively. The operative spectral regions are: $500-758 \mathrm{~nm}$ for $L R$ and $677-780 \mathrm{~nm}$ for HR. It will have a sampling of $0.1 \mathrm{~nm}$ in the oxygen bands (759-769 nm and 686-697 nm) and 0.5-2.0 nm in the red edge, chlorophyll absorption and PRI (Photochemical Reflectance Index) bands. The overlapped spectral range has been introduced to simplify the interband co-registration between the two spectrometers.

Earth radiation is focused by a telescope onto the slit plane with $(F \#=3.1)$. The double slit assembly is placed at the focus plane of the telescope (intermediate focus of the instrument).

A schematic of the optical architecture of the FLEX instrument, and the place of the Slit Assembly within is depicted in the FLEX optical layout in Figure 1.
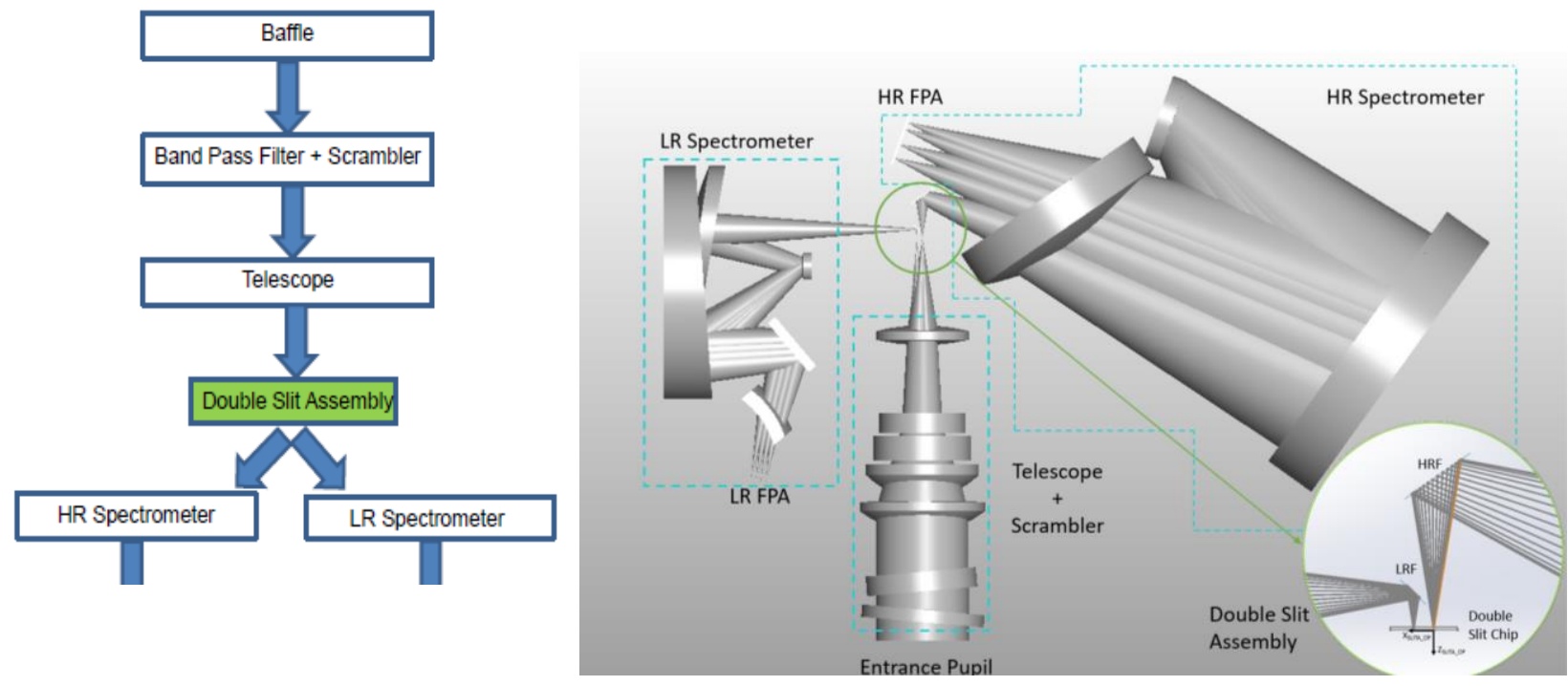

Figure 1: Schematic optical block diagram of FLEX and FLEX Optical Layout. 


\section{SLIT ASSEMBLY - DESIGN}

The main purposes of the Slit Assembly are:

- defining the entrance apertures of the two FLEX spectrometer channels (LR and HR, respectively) by means of two separate slits (double slit)

- spatial separation of the channels' optical paths behind the slits (folding mirrors)

- spectral bandpass filtering of the light

- suppressing any kind of in-field straylight

\section{Description of double slit assembly functional design}

The functional design of the Slit Assembly (SLITA) is presented in Figure 2 SLITA is a passive subsystem in terms of functions. Its main function is the separation of the radiation transmitted by the telescope assembly to the two spectrometer channels LR and HR. Structural, thermal and mounting functions are considered as support functions to the optical one.

The Slit Assembly's main components are a double slit, two plane folding mirrors (HR Fold Mirror and LR Fold Mirror) and mounting structures (Upper and lower Polygon, Base, Double Slit Base) for these elements (see Figure 3).

The double slits ( $44.1 \mathrm{~mm} \times 0.084 \mathrm{~mm}$ each) are separated by $4.5 \mathrm{~mm}$ and must be accurately manufactured and registered with respect to each other.

Shortly behind the double slit, two folding mirrors (HR Fold Mirror and LR Fold Mirror, resp.) deflect the respective optical beams in two 'opposite' directions. Thus, HRS and LRS optical paths are separated. Both mirrors are equipped with very steep optical band-pass filter coatings, which suppress out-of-band radiation and thus in-field stray light. At several positions, dedicated, geometrical structures coated with black diffuse coatings contribute to suppression of out-of-field stray light.

The mechanical mount of the double slit device to the slit assembly (SLITA) is realized via mechanical flexures. They compensate for thermal expansion effect between aluminum structure (SLITA side) and Invar/silicon on double slit device side, respectively (see below).

The SLITA structure is composed of precisely manufactured parts and is made from aluminum, as the two mirrors are. Therefore, thermal effects to mirrors are minimized. The structure is made from several individual parts (Figure 3), which are composed without alignment, since their mutual interfaces are machined highly precise during ultra-precise diamond-machining processes.

The Slit Assembly interfaces to the optical bench of the instrument via the Base part, which also contains several reference surfaces for optical metrology. These surfaces are used to verify the geometrical stability under environmental loads. The stability requirements - on the order of few microns and arc seconds, respectively - are achieved by a combination of opto-mechanical verification tools. 


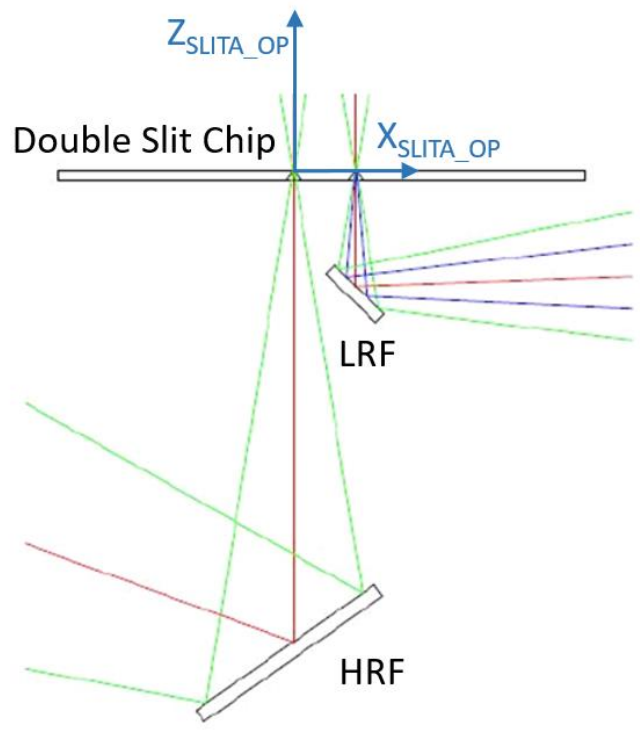

Figure 2: Concept drawing SLITA

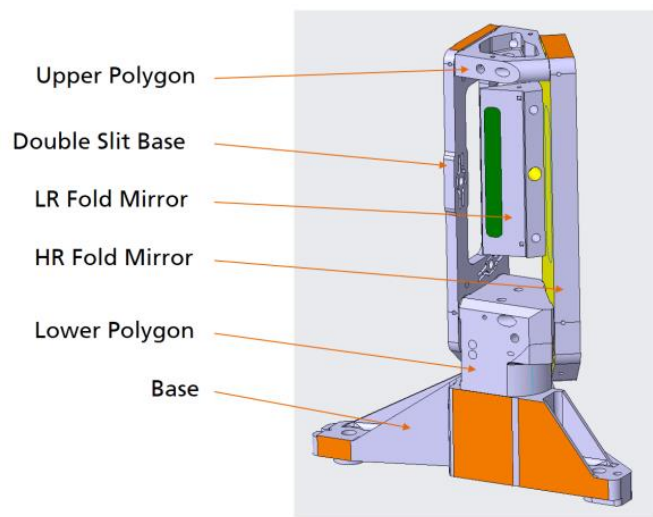

Figure 3: CAD-Design of Slit Assembly (SLITA)

Figure 2 depicts a concept drawing of the optical layout, comprising double slit and the two folding mirrors LRF. Mounting hardware is not shown. Light is focused by telescope onto slit plane. For each channel (HR and LR), after passing the respective slit, light is deflected by a mirror and then enters the corresponding spectrometer (HRS and LRS, respectively). 


\section{DOUBLE SLIT DEVICE}

\subsection{Requirements to Double Slit Device (SLIDS)}

The geometrical requirements of Double slit device are the slit width of $84 \mu \mathrm{m}$ and $44.1 \mathrm{~mm}$ in length for each slit. The tolerances are $2 \mu \mathrm{m}$ in width and $50 \mu \mathrm{m}$ in length, but the positions of the slits to each other and straightness of each slit are required with accuracies of less than $3 \mu \mathrm{m}$. The slit thicknesses must be less than $15 \mu \mathrm{m}$. The required slit planarity of less than $10 \mu \mathrm{m}$ is challenging.

The interface requirements define an absolute positioning of the slits to mechanical interfaces of about $50 \mu \mathrm{m}$ along slit length and width, but 2 arcmin along the rotational degrees of freedom (e.g. optical axis). The knowledge of slit position of $1.5 \mu \mathrm{m}$ for linear and 10 arcsec for rotational measurements are challenging requirements for double slit device characterization.

The environmental loads were driving the design and analysis activities, because there are high shock loads (636 $\mathrm{g}$ at $725 \mathrm{~Hz}$ up to $10 \mathrm{kHz}$ - tolerance $-0 /+6 \mathrm{~dB}$ ). The required ground to orbit stability of less than $5 \mu \mathrm{m}$ of slits with respect to the mechanical interfaces was a challenging one during SLIDS breadboard testing.

\subsection{Design of Double Slit Device}

The double slit device (SLIDS) consists of a double slit upper frame and a double slit lower frame. The double slit chip will be clamped in between the upper and the lower frame. The surface of the lower frame is lapped to reach a planarity of less than $1 \mu \mathrm{m}$. The double slit chip will be planarized by pressing the double slit chip onto a reference plate of the lower frame using mechanical springs (double slit springs). The spring forces guarantee the planarization and the mechanical stable fixation of the double slit chip with respect to mechanical loads. The double slit springs are mounted into grooves of the double slit lower frame. The double slit chip will be additional fixed with a dot of two-component epoxy adhesive to reach the double slit position stability to thermal loads.

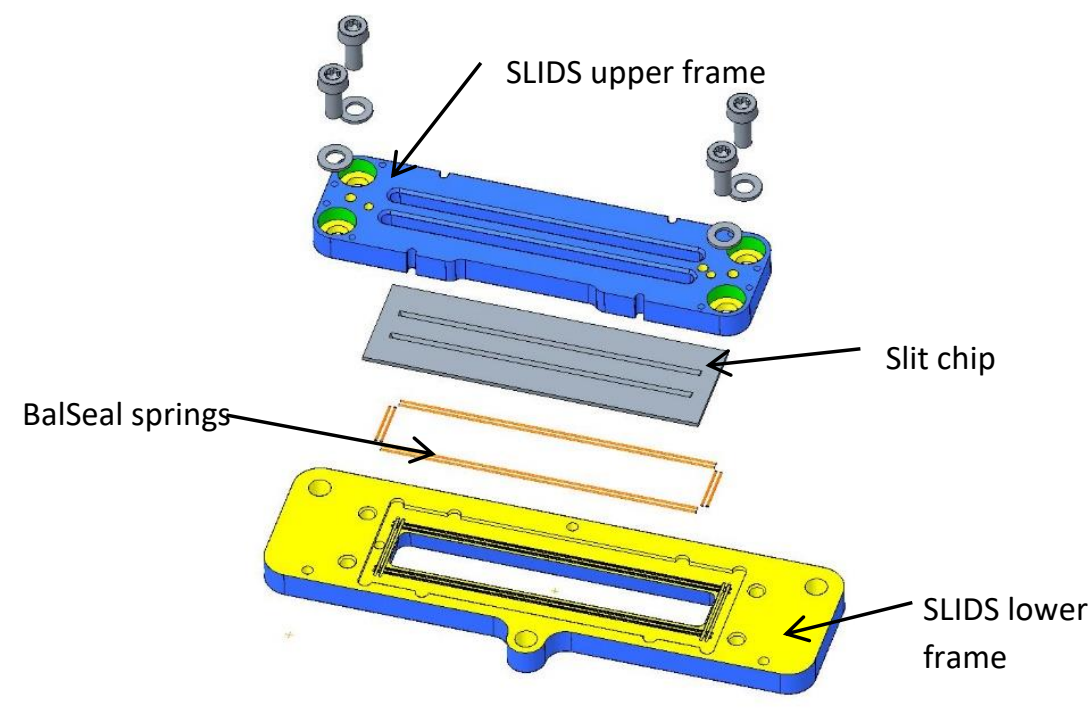

Figure 4: Design of double slit device. 
The material of the double slit upper and lower frame was chosen to Invar to minimize the CTE mismatch of the components - silicon at double slit chip and the frames. The Invar parts will be plated with Nickel-Phosphor as anticorrosive plating. Additionally, those parts will be black-coated by Acktar Magic-Black for minimizing straylight effects.

\subsection{Manufacturing of Double Slit Assembly}

\section{Manufacturing of slit Chip}

The fabrication of the slit structure has been done by a lithographic exposure processes in combination with anisotropic selective Silicon wet-etching processes. The structure definition was performed by electron-beam lithography ensuring highest accuracy and resolution to comply with geometrical requirements.

The structuring process started from one side of a $<100>$ Silicon wafer only. The overall lithographic process flow is shown in Figure 5. A cross section of the slit chip is shown in Figure 6:

1. Resist exposure, lithography

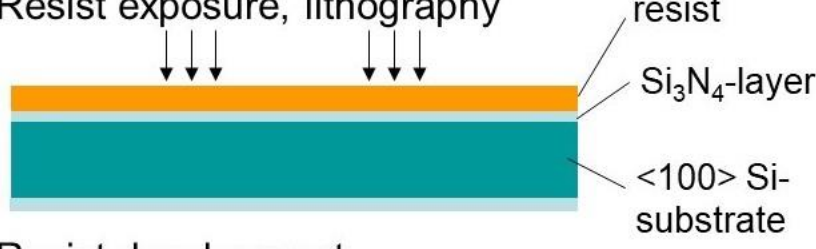

2. Resist development

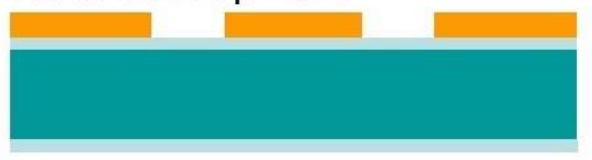

3. Etching of $\mathrm{Si}_{3} \mathrm{~N}_{4}$-layer

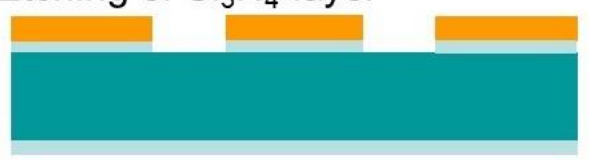

4. Wet-etching of Si substrate

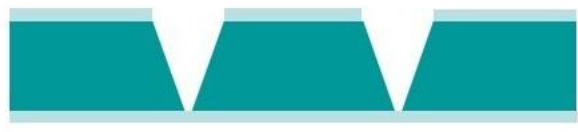

5. Removal of $\mathrm{Si}_{3} \mathrm{~N}_{4}$ layers

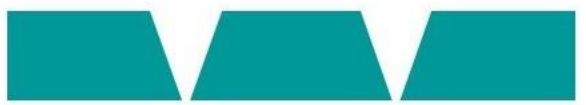

6. Black coating

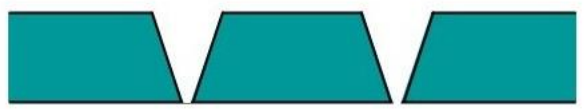

Figure 5: Manufacturing baseline sequence for the realization of the slits.

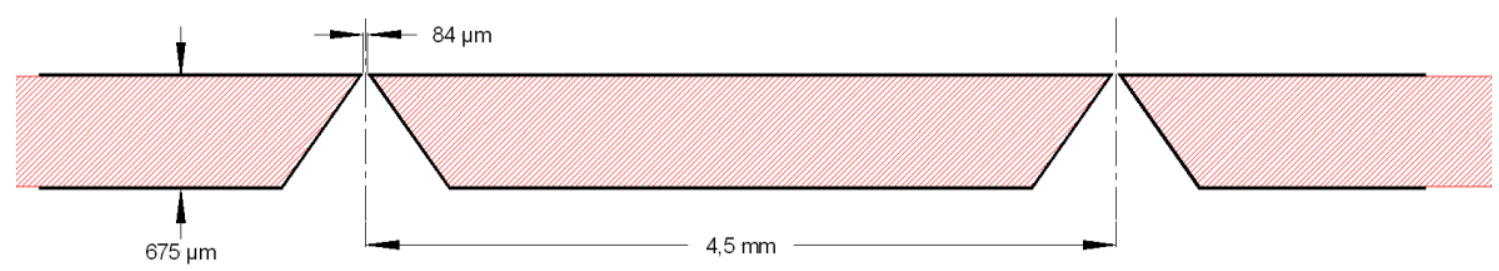

Figure 6: Cross-sectional sketch of the double slit diaphragm with geometric specification 


\section{Black coating on slit chip}

To achieve the reduced reflectivity, both sides of the slit substrate were coated with an Acktar Magic Black layer.

The application of the Magic Black coating was done by Acktar Advanced Coatings. The slits were covered by masks during the coating process. Investigations on Acktar Magic Black coatings inside the clear aperture of the slits were used to determine the offset the slit width including the Acktar coating tolerances. The measurements show a decrease of slit width of $<4 \mu \mathrm{m}$ after black coating application. The slit widths were measured in $500 \mu \mathrm{m}$ steps along the slit length of $44 \mathrm{~mm}$ and in $1 \mu \mathrm{m}$ steps along $168 \mu \mathrm{m}$ according to the specification of the slit width uniformity.

The slit width measurements were made using the Vistec LION LV1 system. The LION is a 20keV e-beam tool with a high precision laser interferometer stage for carrying up to $150 \mathrm{~mm}$ sized substrates and the slit-width measurements are based on pattern edge detection implemented in the software.
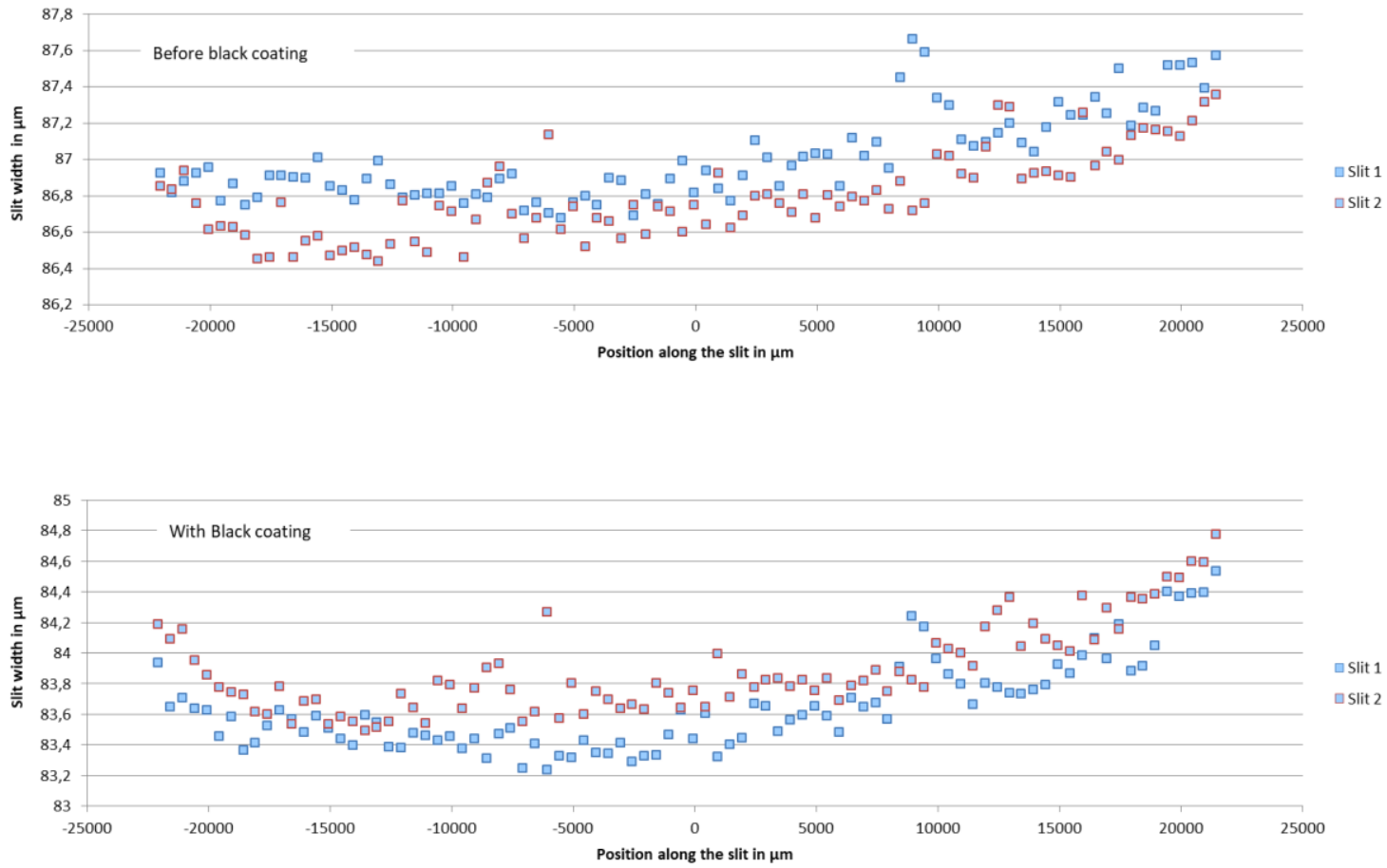

Figure 7: slit width measurements before and after black coating

The averaged total hemispherical reflectance of $1.2 \%$ was reached within a wavelength range from $400 \mathrm{~nm}$ to $1050 \mathrm{~nm}$ on polished silicon substrates of the slit chips. 


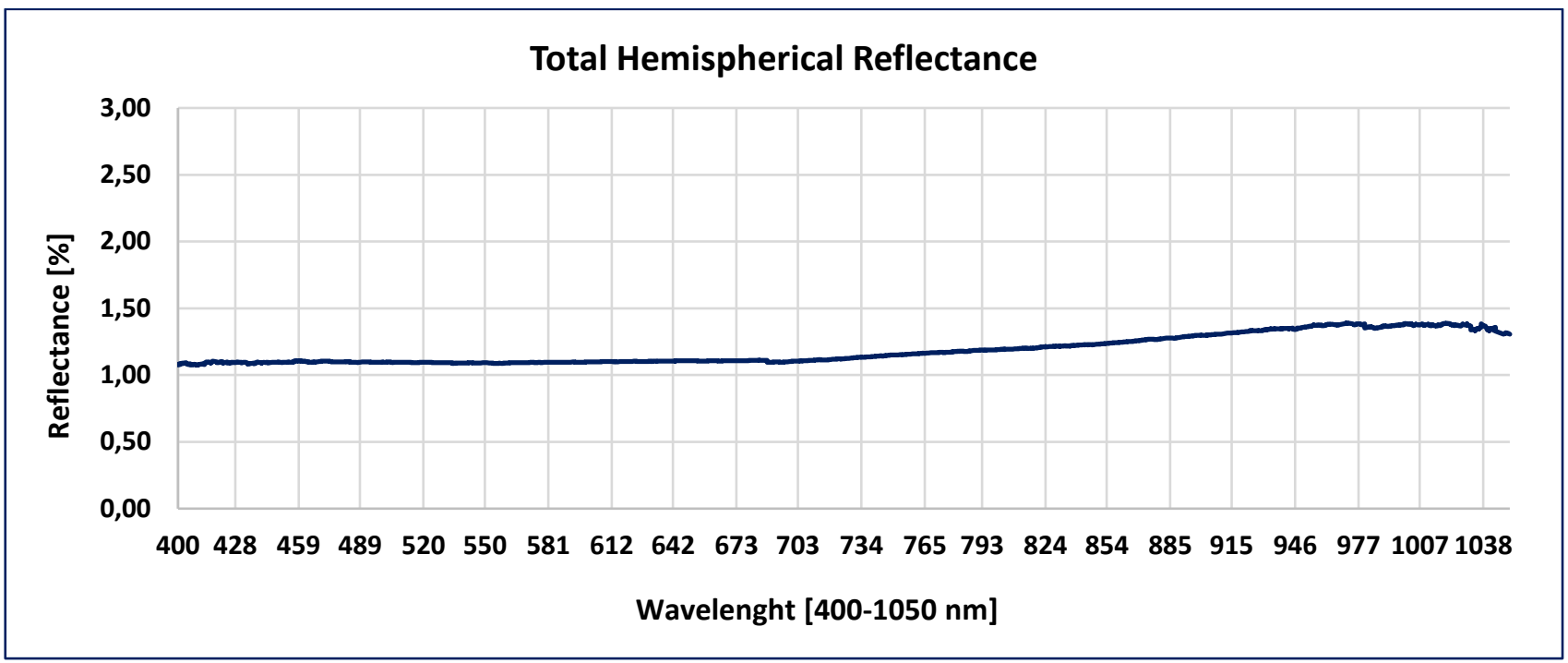

Figure 8: Total hemispherical reflectance of black coatings on slit chips

Edge radii of less than $1 \mu \mathrm{m}$ were reached inside the slits clear apertures. Figure 9 show the surface of Acktar Magic Black coating. The required surface roughness of less than $1 \mu \mathrm{m}$ and the sharp edge radii were reached.

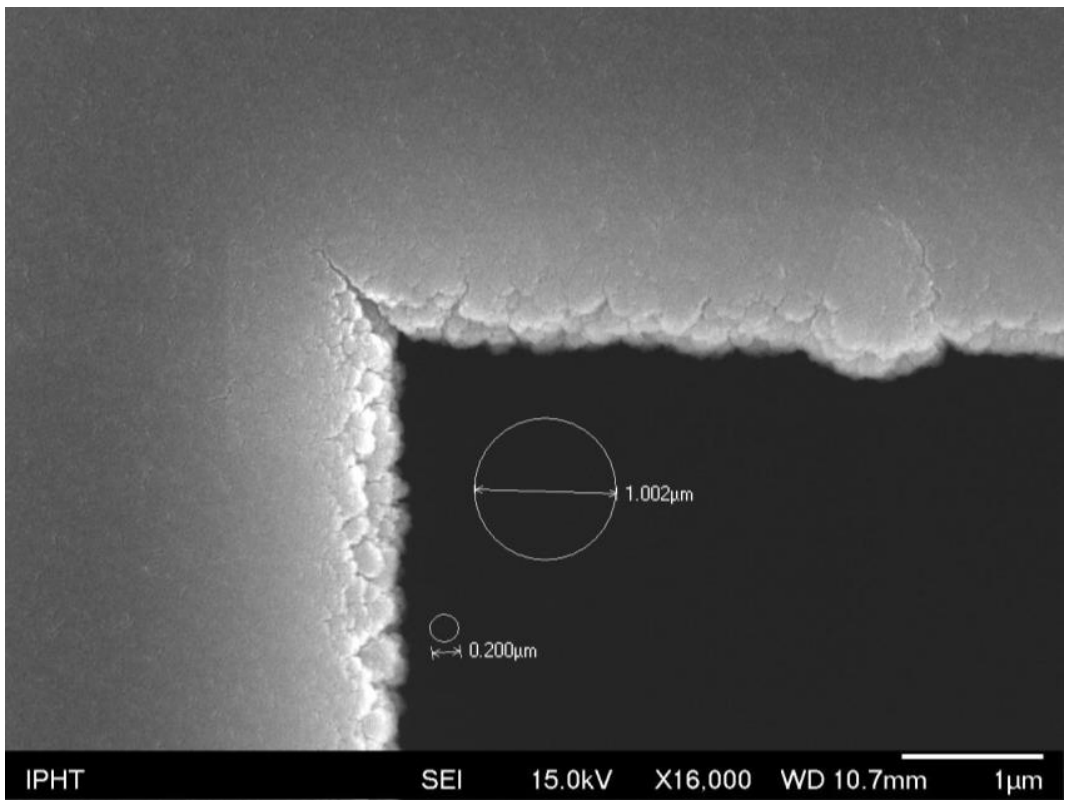

Figure 9: Image on edge radius of slit chip 


\section{Manufacturing of mechanical parts}

The manufacturing of mechanical parts of the double slit device, the upper and the lower frame, was made by milling. During several manufacturing steps, the final geometries of mechanical parts were built. In between the manufacturing steps thermal treatment processes were performed to reduce the relaxation processes to meet the required manufacturing tolerances. The material Invar needs a passivation plating by Nickel-Phosphor. The interface surfaces and the surface of lower frame, which are necessary for slit chip planarization were lapped. A peak to valley planarity of less than $1 \mu \mathrm{m}$ is required to minimize the stress induced deformation to the slit chip and to reach the slits planarity of less than $10 \mu \mathrm{m}$.
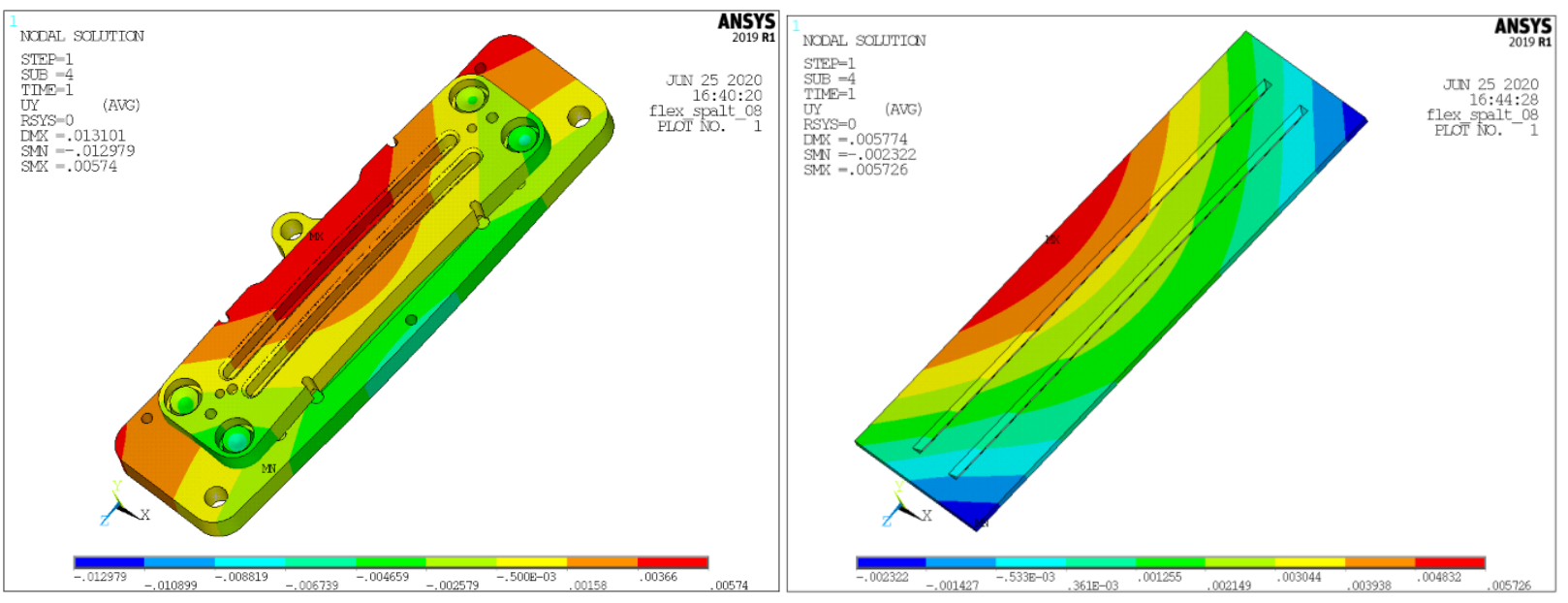

Figure 10: Out-of-plane deformation of the upper and lower frame (left) and the chip (right).

The maximum of the corresponding out-of-plane deformation of the slits chip amounts to $5.7 \mu \mathrm{m}$ (see Figure 10) while a maximum out of plane deformation of $3.5 \mu \mathrm{m}$ occurs at the slit edges induced by the loads by spring forces and a surface planarity of less than $1 \mu \mathrm{m}$.
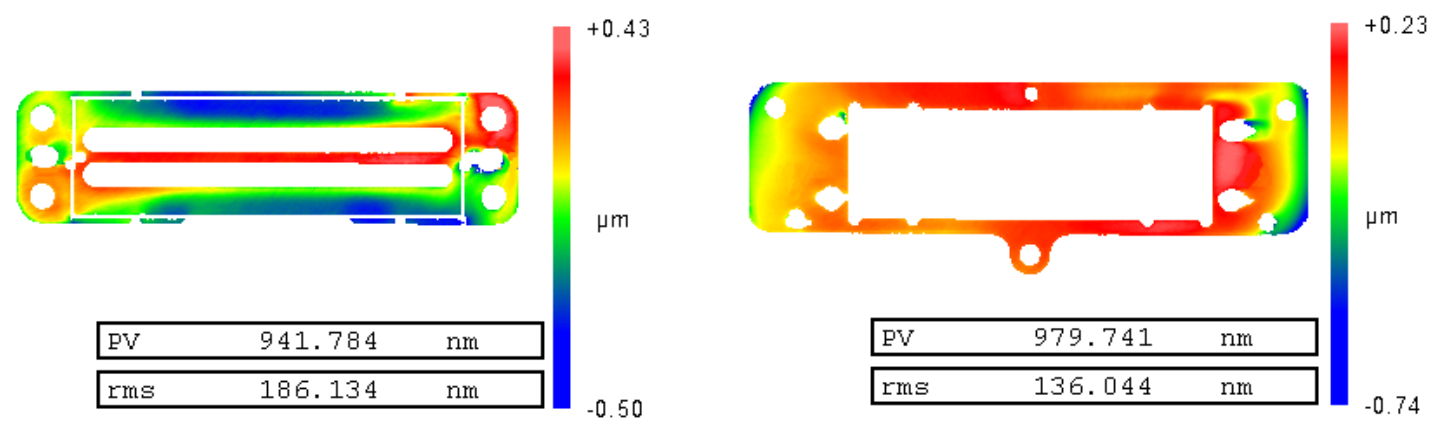

Figure 11 - The interface planarities of SLIDS upper (left) and lower (right) frame. The PV-Values amount to $<1$ micron (PV over common zone) 


\section{Assembly and Integration of Double Slit Device}

The positioning of slit chip to mechanical interfaces is the most challenging task during assembly and integration of double slit device. Mounting and positioning tools were developed for mounting and positioning of upper and lower frame and slit chip. The integration and positioning concept use mechanical references of GSE for slit chip alignment. At first, the upper frame is integrated into the mounting tool. The position of upper frame will be measured and used for positioning of slit chip and lower frame. Adjustable alignment pins will define the position of slit chip and lower frame. After 1-2 iterations the slit chip will be on the required position with respect to the mechanical interfaces of lower frame. The final position will be measured by contact-less optical coordinate measurement. The required positioning accuracy of HRF slit to mechanical interfaces of less than $50 \mu \mathrm{m}$ in $\mathrm{x}-\mathrm{y}$-coordinate and $2 \mathrm{arcmin}$ in $\mathrm{Rz}$ was reached. If the characterization of slit chip position shows the specified values the slit chip will be fixed by a dot of two-component adhesive, applicated onto the upper frame and the slit chip.

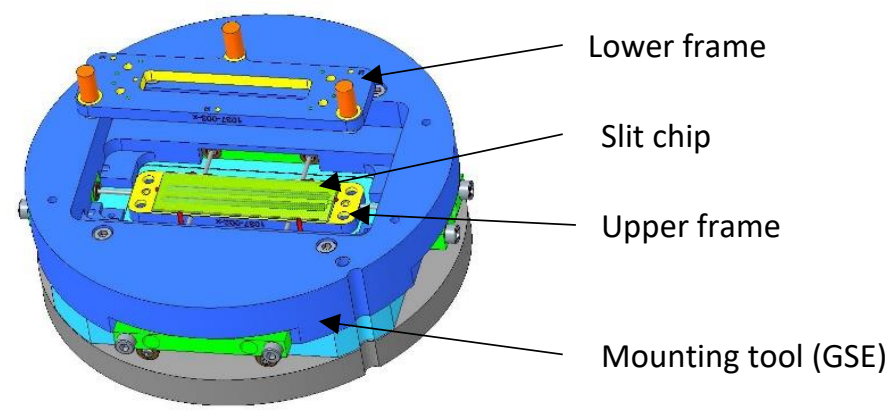

Figure 12: CAD image of assembly tool

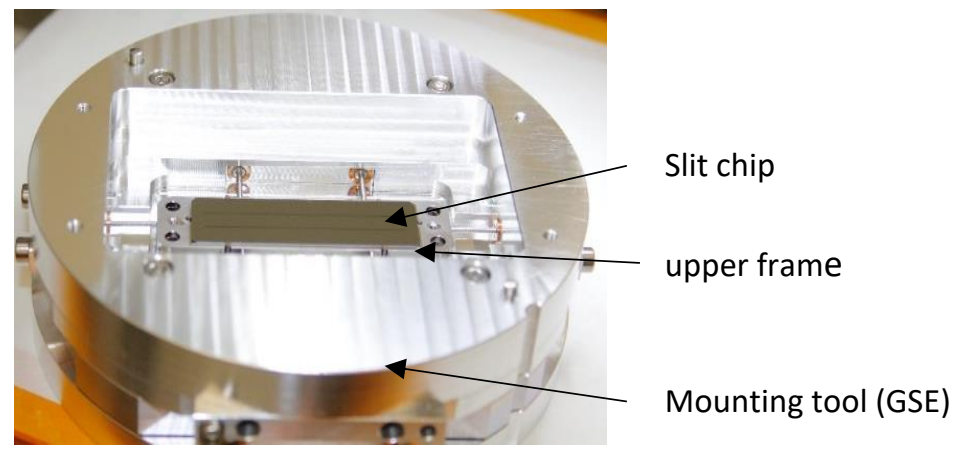

Figure 13: assembly tool with integrated upper frame and slit chip 


\subsection{Test of Double Slit Device}

Because of the high mechanical shock loads of $636 \mathrm{~g}(-0 /+6 \mathrm{~dB})$ the test on double slit device breadboards were performed to prove the slit chip design and the slit chip manufacturing process chain. In an early phase a double slit device was built with a representative slit chip without black coating. The initial shock test shows no damage on the slit chip. A thermal vacuum cycling test was performed on the SLIDS-Breadboard with black coated slit chip. The shock test performed on black coated slit chip shows also no damage. For proofing the design a sine and random vibration test was performed on the uncoated double slit device.

For all tests, the built breadboards of double slit devices were used. The double slit base was manufactured representative to flight hardware. The mechanical parts of base, HR Fold mirror, LR Fold mirror and upper and lower polygons were machined as a structural dummy.

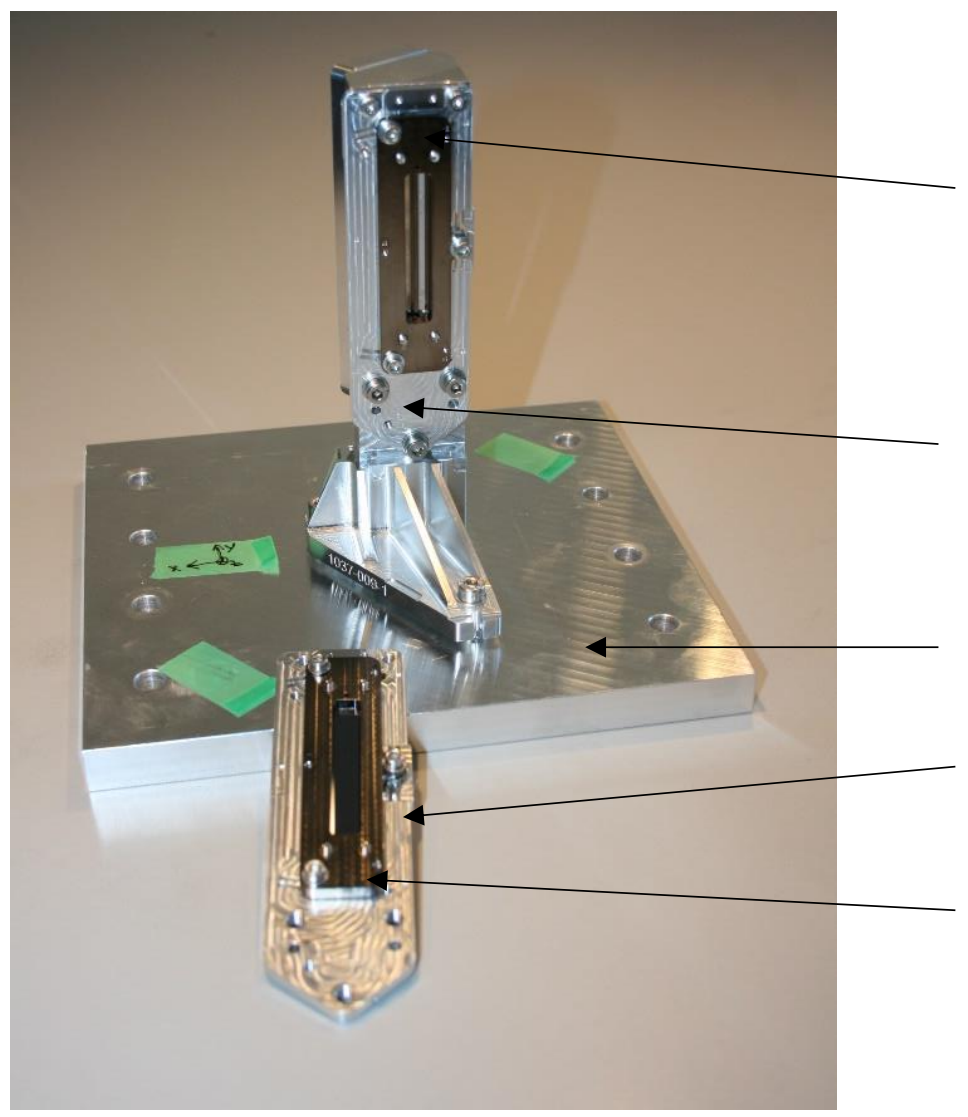

SLIDS-Breadboard

Double slit base

Shock test interface plate

Double slit base

SLIDS-Breadboard

Figure 14: SLIDS on shock test adapter 


\section{Thermal Vacuum Cycle -Test of Double Slit Device}

The SLIDS is flight representative with the exception that the mechanical parts upper and lower frame are not black coated. The resulting temperature range for testing was $-25^{\circ} \mathrm{C}$ up to $+65^{\circ} \mathrm{C}$.

The pressure during test was: $p<1 \times 10^{-5} \mathrm{hPa}$. Before the thermal vacuum test a bake out procedure was performed. The pressure during bake out was: $\mathrm{p}<1 \times 10^{-5} \mathrm{hPa}$. The bake out temperature was $65^{\circ} \mathrm{C}$. The bake out was performed for 12 hours. The thermal cycles during test were performed as given below:

- 25 cycles

- heating to $65^{\circ} \mathrm{C}$ with max. $1 \mathrm{~K} / \mathrm{min}$

- dwell time on $65^{\circ} \mathrm{C}$ for $15 \mathrm{~min}$

- cooling to $-25^{\circ} \mathrm{C}$ with max. $1 \mathrm{~K} / \mathrm{min}$

- dwell time on $-25^{\circ} \mathrm{C}$ for $15 \mathrm{~min}$

- heating to room temperature with max. $1 \mathrm{~K} / \mathrm{min}$

The SLIDS (grey part) was integrated into the thermal vacuum chamber on a thermal conductive base plate (green part) made of Aluminum) see Figure 15.
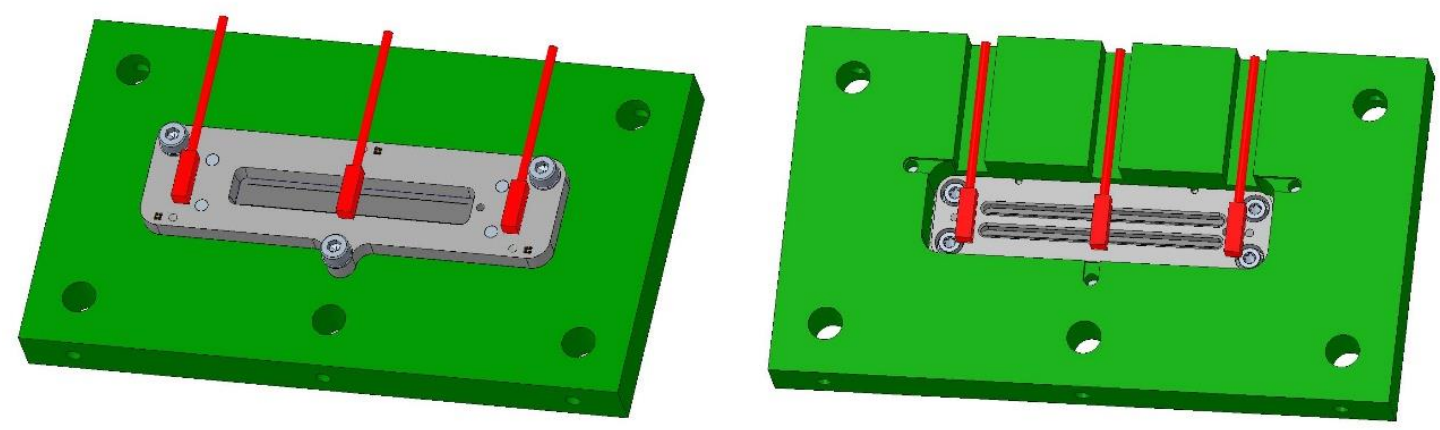

Figure 15: SLIDS on thermal adapter - front and rear side

Up to three temperature sensors (red parts) were mounted onto the SLIDS-front and rear surfaces.

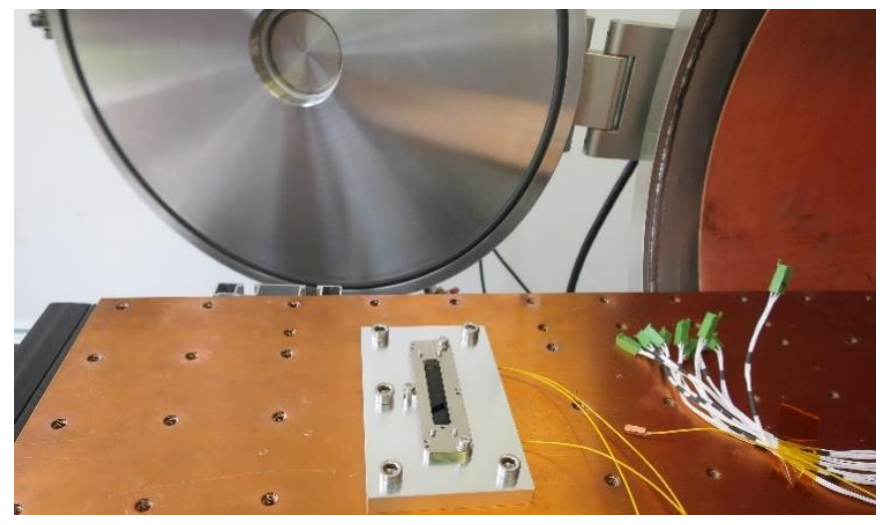

Figure 16: SLIDS on thermal adapter of thermal vacuum chamber 


\section{Shock Test of Double Slit Device}

The shock test was performed along $x-, y$ - and $z$-axis. Per each axis three shocks were applied. For testing the shock setup a SLIDS - dummy without an integrated slit-chip was used. The structure of upper and lower polygon, HRF and LRF was realized by a structural dummy. The SLIDS-base and the SLITA-base was manufactured as flight representative SLITA parts. The black coatings on SLIDS-mechanical parts upper and lower frame were not foreseen. The SLIDS for shock tests were flight representative, the used screws and torques too.
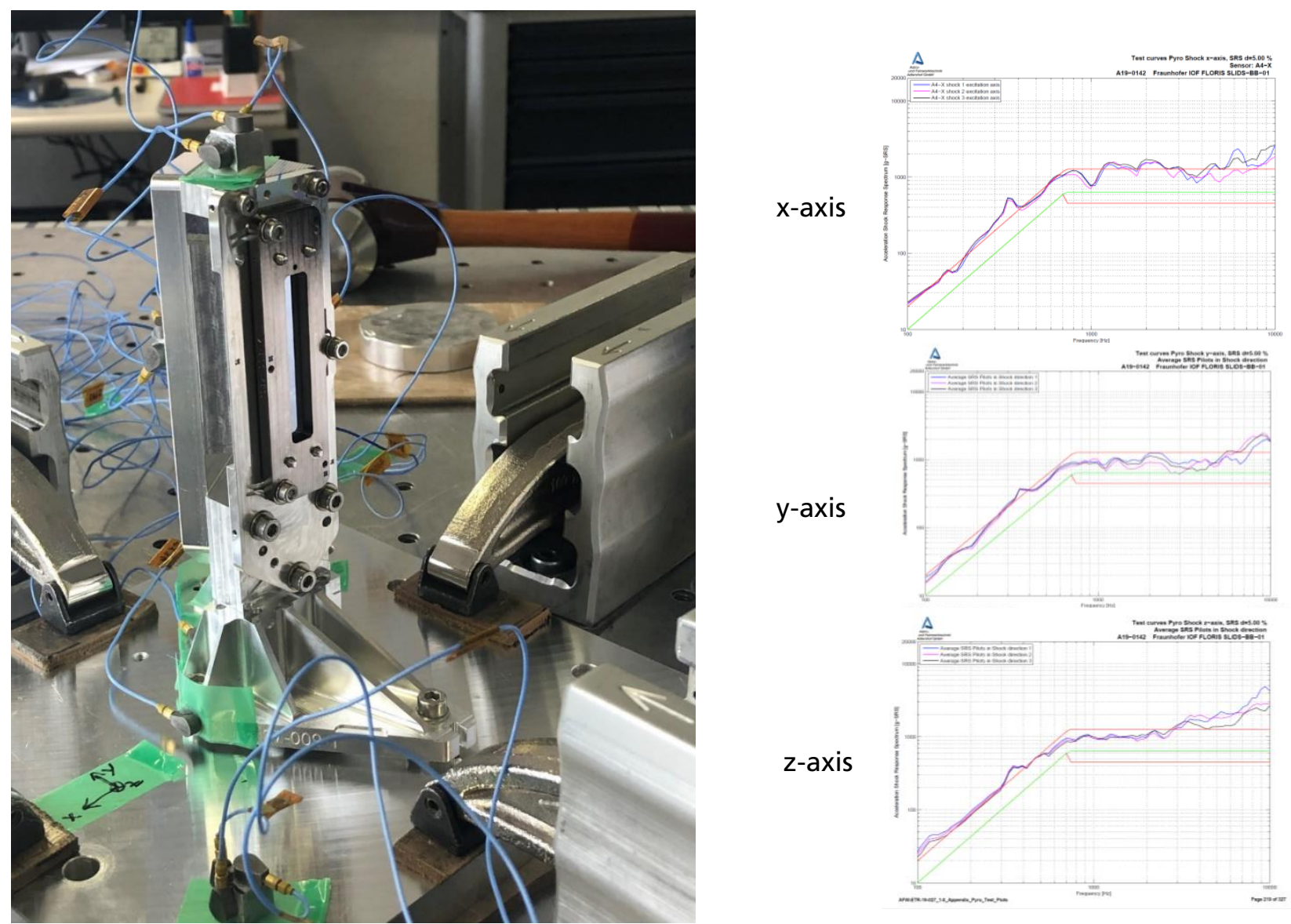

Figure 17: left: SLIDS during shock test (z-axis) - right: SPS shock levels on each axis at interface position

After shock tests the double slit device (SLIDS breadboard) were characterized and show no damage and no position deviations. Thus the manufacturing of slit chips were released on early stage. The shock test of black coated slit chip showed no damages and/or fractures. 


\section{Vibration Test of Double Slit Device}

The sine vibration loads requirements are given in Table 1 and the random vibration loads requirements are given in Table 2.

Table 1: sine vibration loads

\begin{tabular}{|c|c|c|c|c|c|}
\hline \multicolumn{3}{|c|}{ Out of Plane (Z_ph axis) } & \multicolumn{3}{|c|}{$\begin{array}{c}\text { In Plane (X_ph axis, Y_ph } \\
\text { axis) }\end{array}$} \\
\hline f & Acc. & Disp. & f & Acc. & Disp. \\
\hline$[\mathrm{Hz}]$ & {$[\mathrm{g}]$} & {$[\mathrm{mm}]$} & {$[\mathrm{Hz}]$} & {$[\mathrm{g}]$} & {$[\mathrm{mm}]$} \\
\hline 5 & 1.0 & 10 & 5 & 1.0 & 10 \\
\hline 20 & 22.0 & - & 20 & 16.0 & - \\
\hline 100 & 22.0 & - & 100 & 16.0 & - \\
\hline
\end{tabular}

Table 2: random vibration loads

\begin{tabular}{|c|c|c|c|}
\hline \multicolumn{2}{|c|}{$\begin{array}{c}\text { Out of Plane (Z_ph } \\
\text { axis) }\end{array}$} & \multicolumn{2}{c|}{$\begin{array}{c}\text { In Plane (X_ph axis, } \\
\text { Y_ph axis) }\end{array}$} \\
\hline $\mathbf{f}$ & PSD & $\mathbf{f}$ & PSD \\
\hline$[\mathrm{Hz}]$ & {$\left[\mathrm{g}^{2} / \mathrm{Hz}\right]$} & {$[\mathrm{mm}]$} & {$\left[\mathrm{g}^{2} / \mathrm{Hz}\right]$} \\
\hline 20 & 0.010 & 20 & 0.006 \\
\hline 100 & 1.250 & 100 & 0.750 \\
\hline 300 & 1.250 & 300 & 0.750 \\
\hline 400 & 0.500 & 400 & 0.500 \\
\hline 550 & 0.500 & 550 & 0.500 \\
\hline 2000 & 0.038 & 2000 & 0.038 \\
\hline GRMS & $\mathbf{2 5 . 2}$ & GRMS & $\mathbf{2 2 . 5}$ \\
\hline
\end{tabular}

The vibration tests were performed along $x-, y$ - and $z$-axis. Per each axis the sine and random vibration loads were applied. Acceleration sensors were mounted onto the SLIDS.

The sine vibration- and random vibration test flow were performed for $x-, y$ - and $z$-axis in the following steps:

\section{Sine vibration test flow}

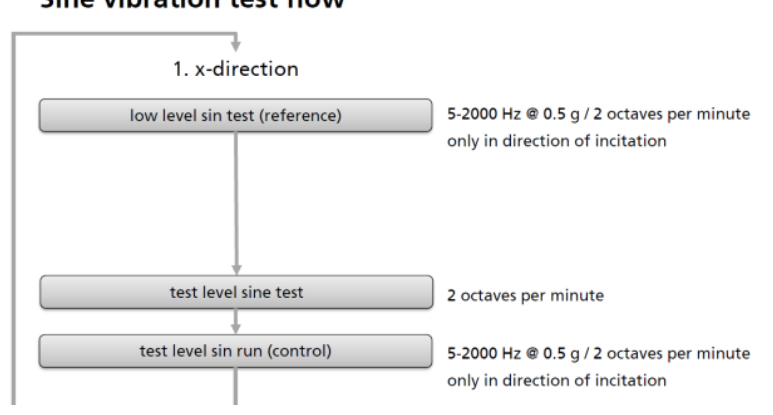

\section{Random vibration test flow}

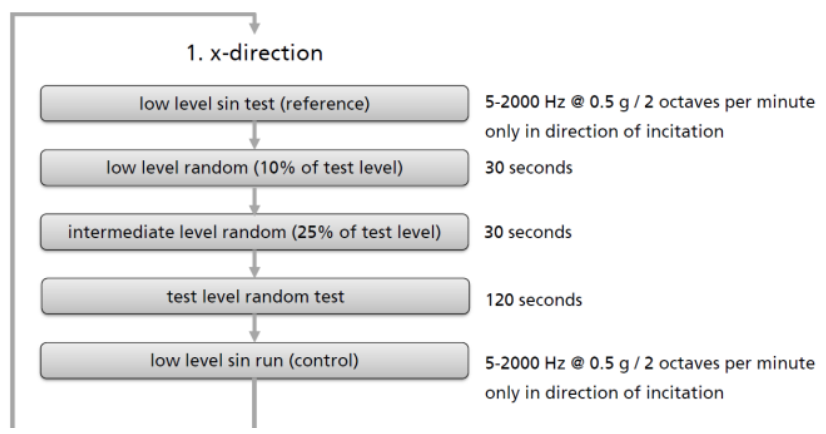

Figure 18: sine vibration test flow

Figure 19: random vibration test flow 


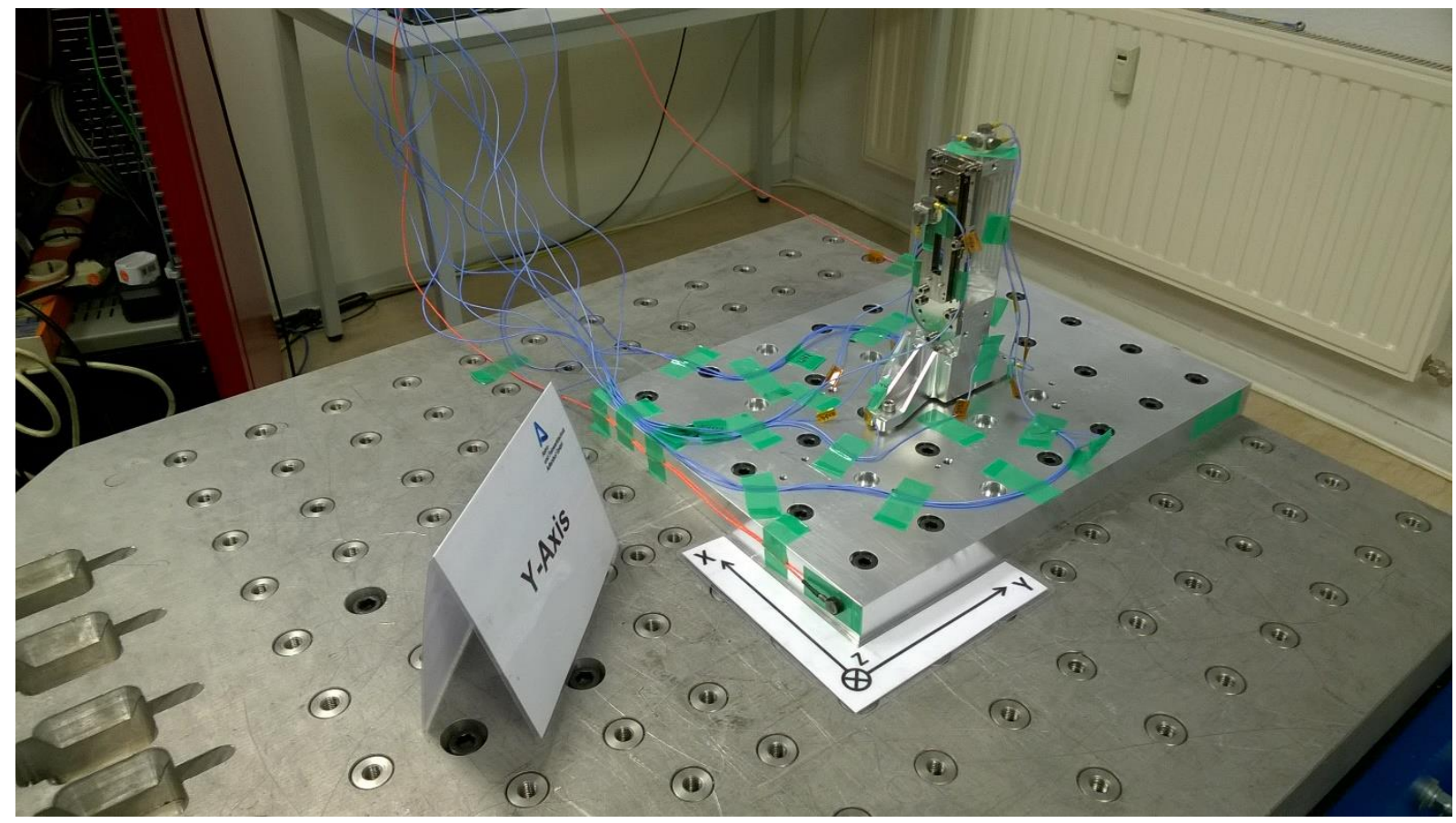

Figure 20: SILDS breadboard on shaker for vibration test - Y-axis

\section{Test results}

After thermal vacuum, shock- and sine- and random vibration tests the double slit devices were characterized. There were no damages, ruptures, cracks and/or fractures of silicon of slit chip visible during inspection using $5 \mathrm{x}$ and $100 \mathrm{x}$ magnification lens. The measurement of slits widths and lengths show no variation. No change of slit planarity $( \pm 1 \mu \mathrm{m}$ tolerance as success criteria) was measured. No position deviations of slit chip position with respect to mechanical interfaces of less than $\pm 1 \mu \mathrm{m}$ were measured. 


\section{CONCLUSION}

The double slit device of the Flex Floris instrument was developed, manufactured, and SLIDS breadboards were successfully tested. The optimized lithographic slit structuring process guarantee the silicon slit chip manufacturing with accuracies in micron-range. The choice of a $<100>$-wafer for double slit structuring was necessary to meet the high mechanical shock loads. The applied black coating on the slit chip also met the requirements of TIS (total integrated scattering), the optical density, and the challenging tolerances of slit width, surface roughness, and edge radii.

The characterization of the tested SLIDS breadboards show full compliance with the geometrical requirements of the slit chip geometry. The slit planarity of less than $10 \mu \mathrm{m}$ were reached after slit chip integration and all tests. The analysis of the SLIDS design with respect to the mechanical and thermal loads show that all environmental requirements were reached. The assembly and integration of SLIDS breadboard was successful. The requirement of slit positioning to mechanical interfaces were also met. The geometrical characterization was performed and confirms the compliance with required geometrical and positioning values.

The thermal vacuum test as well as the vibration and shock test showed no damage of the slit chip and no deviation of slit chip position. Thus, also compliance with ground-to-orbit stability requirements were fully met as was shown as a result of SLIDS breadboard testing.

\section{ACKNOWLEDGEMENTS}

The FLEX Slit Assembly was developed with financial and technical support from the European Space Agency (ESA) in the frame of the Earth Explorer - Fluorescence Explorer (FLEX) mission. We acknowledge support from FLEX team of LEONARDO, Thales Alenia Space (F) and ESA.

\section{REFERENCES}

[1] ESA FLEX website, (https://earth.esa.int/eogateway/missions/flex) (20 January 2021) 